\title{
Encephalitis of Unknown Etiology with Anti-GluR ع2 Autoantibody, Showing Divergent Neuroradiologic and Clinical Findings
}

\author{
Masaki Miyazaki $^{\mathrm{a}}$ Aihide Yoshino $^{\mathrm{a}}$ Toshiya Teraishi $^{\mathrm{a}}$ Soichiro Nomura ${ }^{\mathrm{a}}$ \\ Hideaki Nemoto $^{b}$ Yukitoshi Takahashic \\ ${ }^{a}$ Department of Psychiatry, National Defense Medical College, Saitama, ${ }^{b}$ Department of Neurology, \\ Kohnodai Hospital, National Center of Neurology and Psychiatry, Chiba, and 'Department of Pediatrics, \\ National Epilepsy Center, Shizuoka Institute of Epilepsy and Neurological Disorders, Shizuoka, Japan
}

Dear Sir,

A patient with severe temporal lobe atrophy, memory disturbance, and personality deterioration caused by encephalitis of unknown etiology was admitted for evaluation. Cerebrospinal fluid (CSF) studies disclosed no virus, but autoantibodies were detected against the N-methyl-D-aspartate-type glutamate receptor epsilon 2 (GluR $\varepsilon 2$ ). GluR $\varepsilon 2$ channels have been implicated in synaptic plasticity and localization associated with neural development and learning [1]. Recently autoantibodies against GluR $\varepsilon 2$ were found in some patients with Rasmussen's encephalitis [2] and nonherpetic limbic encephalitis [3], suggesting an autoimmune pathogenesis for some encephalitis. Surprisingly in our case, neuropsychiatric symptoms did not worsen when progressively severe neuroradiologic alterations in the temporal lobes appeared after hospitalization.

\section{Case Report}

A 36-year-old man was admitted to our hospital for evaluation concerning the etiology of severe memory impairment. Neither he nor family members had a prior history of epilepsy, dementia, autoimmune diseases, or neuropsychiatric disorders. He had no previous history of alcohol and/ or drug abuse. He had been diagnosed with diabetes 2 years previously, but was lost to follow-up before any treatment. He had had a headache which lasted for several days 5 months before admission to our hospital. A short time later he developed severe memory impairment and pathologically increased appetite; he repeatedly stole food, even eating pet foods.

Table 1 presents laboratory, neuropsychologic, electroencephalographic, and neuroradiologic findings over time. Biochemical examinations were normal except for a serum glucose concentration of $320 \mathrm{mg} / \mathrm{dl}$ and a glycosylated hemoglobin (HbAlc) value of $15.7 \%$. Antibodies in the serum for HIV and syphilis were negative. The Wechsler Adult Intelligence Scale-Revised (WAIS-R) showed a verbal intelligence quotient (VIQ) of 85, a performance IQ (PIQ) of 85, and a full scale IQ (FIQ) of 84. The Wechsler Memory Scale-Revised (WMS-R) showed very poor general memory, visual memory, verbal memory, and delayed-recall memory (scores of $<50,64$, $<50$, and $<50$, respectively), while the score on the attention-concentration scale was within the normal range (a score of 106). Electroencephalography (EEG) showed normal background activity and no epileptic discharges. Routine CSF study showed no abnormalities except for moderate elevation of protein $(60 \mathrm{mg} / \mathrm{dl}$; normal range 10-40). CSF cell count was $3 / \mathrm{mm}^{3}$ (normal range $1-6$ ).

MRI of the brain demonstrated severe diffuse cerebral atrophy, with accentuation in the mesial temporal lobes including the hippocampi (fig. 1A), which was suggestive of progressive dementia. However, repeat MRI (fig. 1B) on the 13th hospital day disclosed a new area of high signal intensity centered at the right superior temporal gyrus, suggesting progressive encephalitis. Despite this striking signal alteration, CSF protein and cell counts were within the normal range on three occasions, days 14,49 , and 88 . Body temperature remained within the normal range throughout the course of illness. Polymerase chain reaction (PCR) did not detect herpes simplex virus (HSV)-1 or 2, human herpes virus- 6 or 7 , cytomegalovirus, or Epstein-Barr virus in the CSF on days 49 or 88 . Systemic radiologic examination did not disclose a malignant neoplasm while anti-Hu antibodies were not detected in the CSF at a dilution of 1:2,000 by Western blotting, probably excluding paraneoplastic limbic encephalitis. Blood sugar remained well below $200 \mathrm{mg} / \mathrm{dl}$.

\section{KARGER}

Fax +41613061234 E-Mail karger@karger.ch www.karger.com www.karger.com/ene
Aihide Yoshino, MD

Department of Psychiatry, National Defense Medical College

3-2 Namiki, Tokorozawa

Saitama 3598513 (Japan)

Tel. +81 42995 1619, Fax +81 42996 5203, E-Mail aihide@ndmc.ac.jp 
Fig. 1. Coronal FLAIR MRI sequences. A Day 4: diffuse cerebral atrophy is seen, especially in the mesial temporal lobes including the hippocampi. B Day 13: a new area of high signal intensity is centered in the right superior temporal gyrus. C Day 46: severe signal alteration with edema affects extensive areas of the temporal lobes. D Day 172: abnormal signal disappeared and brain atrophy remained the same as on day $4(\mathbf{A})$.
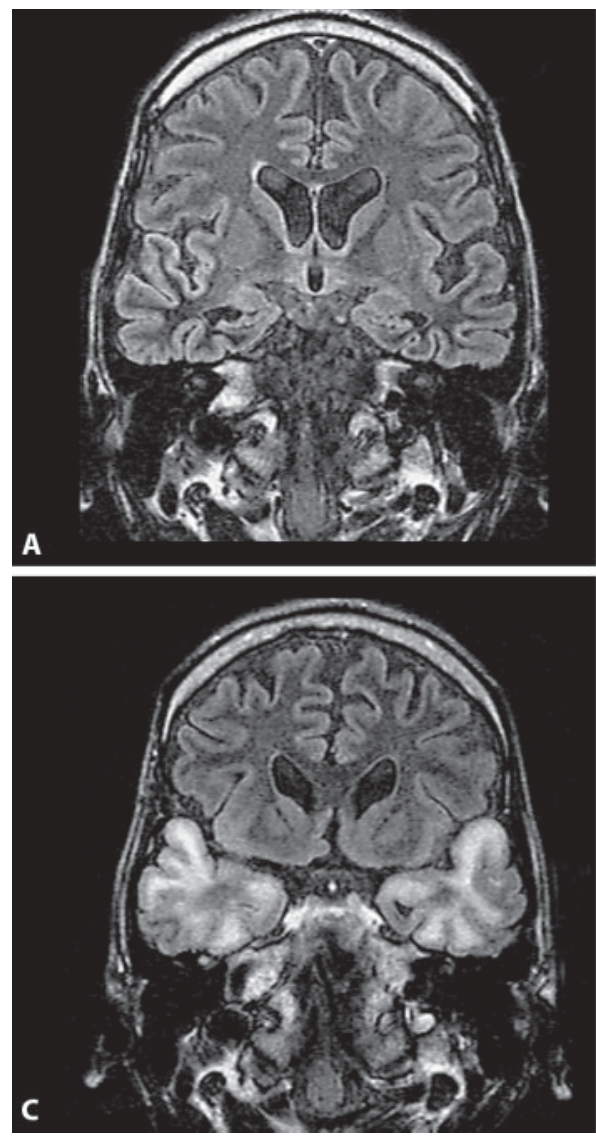
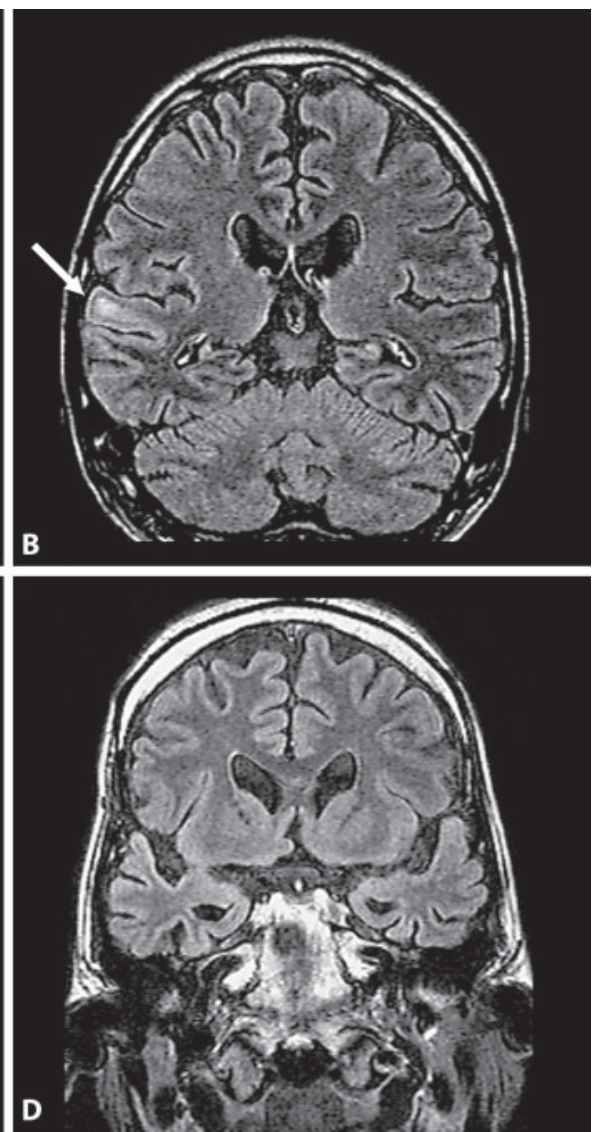

Table 1. Laboratory, clinical, and neuroradiologic course

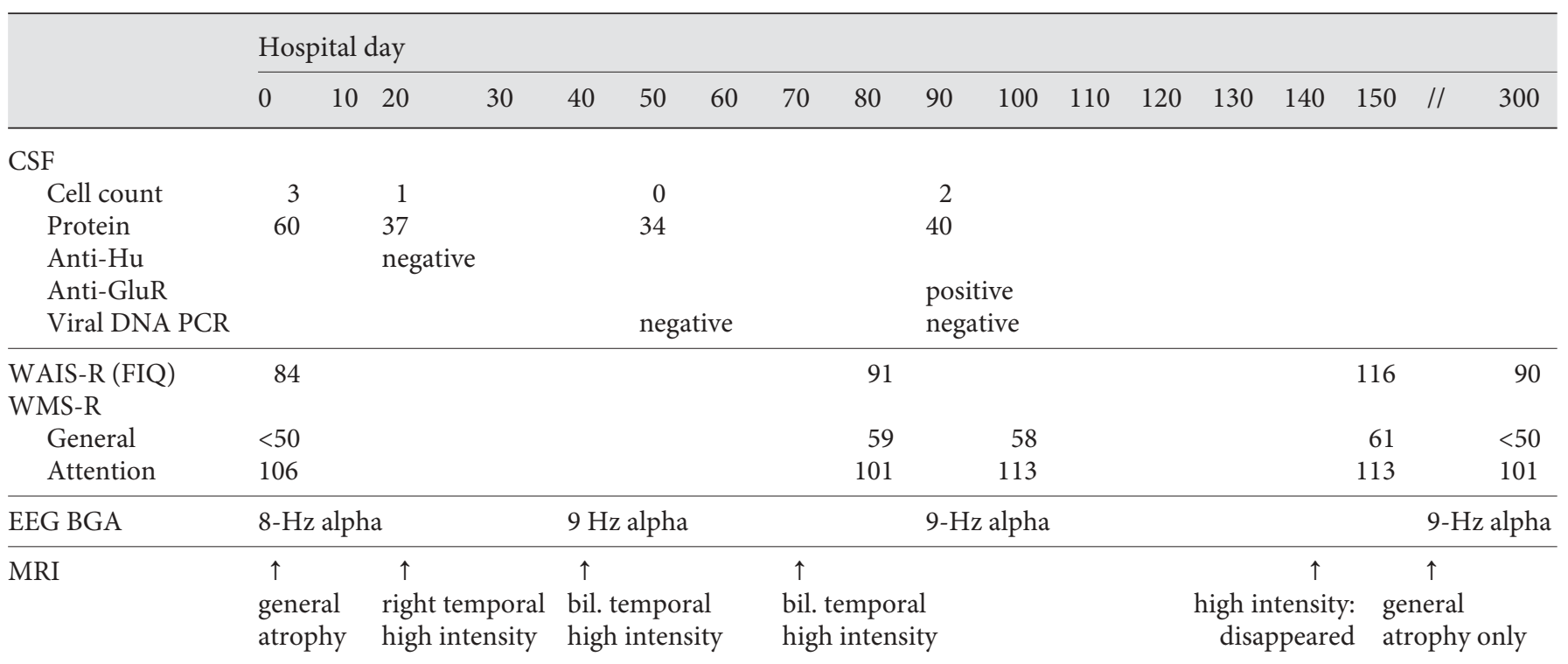

$\mathrm{BGA}=$ Background activity; bil. = bilateral. 
On day 46, MRI showed that the severe signal alteration with edema had extended to wide areas of the temporallobes (fig. 1C). Diffusion-weighted image (DWI) also demonstrated new areas of high intensity at the temporal lobes. Moreover, ${ }^{99 \mathrm{~m}} \mathrm{Tc}$ single-photon emission computed tomography (SPECT) showed significant hyperperfusion in mesial and lateral portions of both temporal lobes, suggesting encephalitis of unknown etiology localized to the temporal lobes. Surprisingly, however, the neuropsychiatric status did not deteriorate, and disturbances of consciousness or epileptic seizures did not occur. On reexamination, WAIS-R and WMS-R showed no significant change (VIQ 85, PIQ 100, FIQ 84, and a score of 101 on the attentionconcentration scale). MRI findings had not improved by day 74 despite 10 days of acyclovir therapy. CSF immunoassay for 14-3-3 protein was negative, suggesting that broad, persistent neural injury was not actively progressing. Repeated EEG showed no abnormalities of background activity or epileptic discharges. The patient did not manifest any epileptic seizures, including epilepsia partialis continua and nonconvulsive status epilepticus. Although we did not examine CSF for myelin basic protein or oligoclonal bands, acute disseminated encephalomyelitis was unlikely since MRI signal alterations were diffuse rather than scattered, and repeated EEGs were within the normal ranges.

CSF obtained on day 88 was then examined for autoantibodies against GluR $\varepsilon 2$, as these have been reported in some patients with nonherpetic limbic encephalitis [3]. Serum and CSF were tested for anti-GluR $\varepsilon 2$ at dilutions of 1:20 and 1:15, respectively. Only CSF IgG, not IgM, autoantibodies were demonstrated. On day 172 , the extent of signal alteration clearly had decreased, and the abnormal signal subsequently disappeared (fig. 1D). Overall brain atrophy remained the same as on admission. SPECT showed the disappearance of hyperperfusion in the temporal lobes; the mesial temporal areas now showed hypoperfusion. A third WAIS-R and WMS-R examination showed no notable change. Symptoms and neuroradiologic findings have remained stable after discharge from the hospital.

\section{Discussion}

We suspected that the patient initially might have had encephalitis of unknown etiology with severe residual memory impairment and marked atrophy of the mesial temporal cortex. We have had no similar clinical experience in which the first insult causing severe memory impairment was not evident as a history of disturbance of consciousness or epileptic seizure. Five months after the presumed first insult, findings indicated an encephalitis of uncertain etiology showing a marked discrepancy between worsening MRI findings and stable clinical status. Although MRI and SPECT showed severe signal alteration with hyperperfusion in the temporal lobes, neuropsychiatric symptoms did not deteriorate. We know of no reported case of encephalitis with such a divergent clinicoradiologic course. A previously reported case of nonherpetic limbic encephalitis in which neuroradiologic findings disappeared after 6 months showed no relapse [4]. Since some relapsing cases of herpetic encephalitis did not have HSV detectable in CSF by PCR [5], we could not exclude the possibility that his first insult represented herpetic encephalitis, but few clinical symptoms characteristic of herpetic encephalitis were present.

Although the patient's illness may have been caused by an undetected infectious agent, we would propose a different explanation: autoimmune encephalitis. A cellmediated immune response may have caused tissue damage during an infectious episode 5 months before admission, re- sulting in production of autoantibodies against GluR $\varepsilon 2$. Alternatively, antibodies developing in response to the infectious agent may later have acted as autoantibodies against GluR $\varepsilon 2$ because of molecular homology.

Apparent clinical stability in the presence of dramatically worsening neuroradiologic findings during hospitalization may have reflected an initial insult before admission so severe that the WAIS-R and WMS-R could not detect a further decline in temporal lobe function during the admission. Although we could not determine whether autoantibodies against GluR $\varepsilon 2$ were the cause or the result of the encephalitis of unknown etiology, we believe that the autoantibodies had a profound influence on the neuroradiologic course.

\section{References}

1 Mori H, Manabe T, Watanabe M, Satoh Y, Suzuki N, Toki S, Nakamura K, Yagi T, Kushiya E, Takahashi T, Inoue Y, Sakimura K, Mishina M: Role of the carboxy-terminal region of the GluR epsilon2 subunit in synaptic localization of the NMDA receptor channel. Neuron 1998;21:571-580.

2 Takahashi Y, Mori H, Mishina M, Watanabe M, Fujiwara T, Shimomura J, Aiba H, Miyajima T, Saito Y, Nezu A, Nishida H, Imai K, Sakaguchi N, Kondo N: Autoantibodies to NMDA receptor in patients with chronic forms of epilepsia partialis continua. Neurology 2003;61:891-896.

3 Mochizuki Y, Mizutani T, Isozaki E, Ohtake T, Takahashi Y: Acute limbic encephalitis: a new entity? Neurosci Lett 2006;394:5-8.

4 Bien CG, Schulze-Bonhage A, Deckert M, Urbach H, Helmstaedter C, Grunwald T, Schaller C, Elger CE: Limbic encephalitis not associated with neoplasm as a cause of temporal lobe epilepsy. Neurology 2000;55: 1823-1828.

5 De Tiege X, Rozenberg F, Des Portes V, Lobut JB, Lebon P, Ponsot G, Heron B: Herpes simplex encephalitis relapses in children: differentiation of two neurologic entities. Neurology 2003;61:241-243. 